\title{
Relación entre movilidad internacional y prejuicio moderno de españoles hacia el colectivo inmigrante en España
}

Mariana Carmona Vescance*

\section{RESUMEN}

Pese a la aceleración de los flujos migratorios a nivel internacional en las últimas décadas, el prejuicio sigue estando presente en las sociedades contemporáneas. En el presente artículo se revisa la relación entre la movilidad internacional y el prejuicio moderno en España. La muestra corresponde a 255 espańoles de nacimiento que se encontraban viviendo en Espańa en el momento de la evaluación, con una edad entre los 18 y 63 años $(M=33,24$; DT $=9,38)$ y una participación femenina del $58,4 \%$. La muestra fue recogida de manera no probabilística mediante el uso de tecnologías de la información. Para estudiar la relación entre movilidad y prejuicio moderno, se construyó un cuestionario de preguntas cerradas. Asimismo, se utilizó la escala de medición de prejuicio de Pettigrew y Meertens (1995) en la adaptación realizada por Frias-Navarro (2009) para la población española. Los análisis estadísticos se llevaron a cabo mediante pruebas de correlación y pruebas ANOvA entre-sujetos unifactorial. Los resultados muestran que a mayores efectos de la movilidad, mayor (y mejor) contacto con inmigrantes, así como menores niveles de prejuicio. Las implicaciones del estudio señalan que dados los resultados de consistencia interna y fiabilidad de la escala construida de los efectos de la movilidad, se le considera un instrumento adecuado para estudiar la relación entre movilidad internacional y prejuicio moderno, de cara a futuras investigaciones.

Palabras clave: efectos de la movilidad, prejuicio moderno, inmigrantes, teoría del contacto intergrupal, capital de movilidad.

\footnotetext{
* Maestría en Migraciones. Consultora de la Fundación Acción Positiva. Bogotá (Colombia). mcarmonavescance@ outlook.com

Recibido: 19 de marzo de 2015 / Modificado: 10 de abril de 2015 / Aceptado: 23 de abril de 2015

Para citar este artículo

Carmona, M. (2015). Relación entre movilidad internacional y prejuicio moderno de españoles hacia el colectivo inmigrante en España. OASIs, 22, 157-175. DOI: http://dx.doi.org/10.18601/16577558.n22.08
} 


\section{The relation between inter- national mobility and the modern prejudice of Spaniards toward the immi- grant collective in Spain}

\section{ABSTRACT}

Although international migration has increased in volume during the last decades, prejudice against immigrants is still present in contemporary societies. In the present article, we study transnational human capital and modern prejudice in Spain. The sample consists of 255 Spaniards (born in Spain) that were living in Spain at the moment of the survey. The age range of the sample is between 18 and 63 years $(\mathrm{M}=33,24 ; \mathrm{SD}=9,38)$ and $58,4 \%$ of them are women. The sample is non-probabilistic and was taken through the Internet. To study the relationship between transnational human capital and modern prejudice, we created a survey with closed questions to measure mobility effects. To measure modern prejudice, we used the instrument created by Pettigrew and Meertens (1995) and adapted by FriasNavarro (2009) to the Spanish population. The statistics analysis was done by correlations and ANOvA. The results show that the more mobility effects, the more (and better) contact with immigrants, and also lower levels of prejudice. The implications of the study are that the measure of the scale of mobility effects is an appropriate instrument to study the relationship between international mobility and modern prejudice, based on the properties, structure and correlates of that scale.
Keywords: Mobility effects, intergroup contact theory, modern prejudice, immigrants, transnational human capital.

\section{INTRODUCCIÓN}

La migración internacional es un fenómeno social caracterizado - entre otros aspectos- por la aceleración cuantitativa de los flujos de personas que traspasan las fronteras de sus países (Castles y Miller, 2009; Dervin y Ljalikova, 2008; Knafou, 1998; Wihtol de Wenden, 2001). El debate sobre la democratización de la migración internacional, i.e. la posibilidad de viajar al alcance de todos, sigue cuestionando la existencia de barreras de entrada y de salida, así como la falta de capital (económico) y la generalización de las políticas restrictivas en los países desarrollados (Glick y Salazar, 2013; Terrier 2009; Wihtol de Wenden 2001).

Los actores locales e internacionales se enfrentan hoy en día a nuevas migraciones y movilidades que exigen un tratamiento diferencial, como son la aparición de los menores no acompañados (Simon, 2008); la feminización de las olas migratorias (Castles y Miller, 2009; Simon, 2008); la movilidad internacional de estudiantes (Erlich, 2012; Murphy-Lejeune, 2001, 2003, 2013; Simon, 2008; Terrier, 2009); la "circulación" de cerebros (Changgui, Rosen y Zweig, 2004); los movimientos circulares/pendulares (Tarrius, 1992; Garneau 2006; Simon, 2008), entre otros.

Según la Organización Internacional para las Migraciones (OIM), el contingente de migrantes internacionales en 2010 fue de 214.200 millones, cifra que "ha permanecido 
relativamente estable como porcentaje de la población mundial [...] entre el 2005 y el 2010" (oIm, 2010, p. 119).

Por su parte, España ha presentado en los últimos diez años una inversión de la tasa neta de migración, al pasar de país emisor a país receptor de personas (Garneau, 2006; Rojas, 2008; Wihtol de Wenden, 2001). Igualmente, el flujo de españoles que emigraron al exterior creció de manera paulatina entre el 2008 y el 2013, al pasar de 34.427 a 71.068 personas.

En lo que respecta al turismo internacional, la Organización Mundial de Turismo (омт) calcula que en el 2012, "la llegada de turistas internacionales creció un 4\% alcanzando los 1.035 millones [..] España ocupa el cuarto lugar [a nivel mundial] por llegadas con 58 millones de personas" (омт, 2013, p. 4).

Los datos relativos a las migraciones y la movilidad por motivos turísticos se presentan para dar cuenta del aumento de la movilidad internacional, entendida esta como el desplazamiento geográfico por fuera del país de origen, sin importar la prolongación de la estancia en el exterior.

Pese a que las migraciones no son una situación nueva (Garneau, 2006), el prejuicio es una actitud todavía presente en las sociedades contemporáneas, el cual ha sido estudiado desde hace varias décadas por diversas disciplinas $\mathrm{y}$ autores, formulando constructos como racismo moderno (McConahay, 1986), racismo aversivo (Dovidio y Gaertner, 1986), teoría integrada de la amenaza (Stephan y Stephan, 2000) y prejuicio moderno, también conocido por sus escalas: manifiesto y sutil (Pettigrew y Meertens, 1995), entre otros.
Dicha evolución teórico-histórica del prejuicio indica que, lejos de ser un tema superado en la sociedad contemporánea, este ha mutado o derivado en nuevas formas de prejuicio, que se presentan de manera más sutil y que están relacionadas con el grado de deseabilidad social percibido por los individuos y sus emociones (Frias, Barrientos, Badenes, Monterde-i-Bort y Cárdenas, 2013; Pettigrew y Meertens, 1995).

De esta forma, el conflicto abierto, frontal y hostil que se mantenía en el pasado entre colectivos poblacionales, ha pasado a tener nuevas características acordes a los cambios históricos de las sociedades, enmarcadas en el discurso políticamente correcto hacia quienes son considerados como diferentes.

Por su parte, el capital de movilidad relativo a las migraciones internacionales hace referencia a los recursos adquiridos por una persona durante su trayectoria vital, que indican la posibilidad de desplazamiento al exterior o su movilidad geográfica real o imaginaria anterior, y que facilitan a su vez la realización de viajes y estancias futuras al extranjero (Agulhon y Xavier de Brito, 2010; Allemand, 2004; Bredeloup, 2008; Chatterji, 2013; Erlich, 2012; Garneau 2006; Murphy-Lejeune, 2001).

Dicho desplazamiento produce efectos en los individuos, en términos de aprendizaje lingüístico, apertura, mayor conocimiento de otras culturas, entre otros (Murphy-Lejeune, 2001, 2013). Se desconocen instrumentos de medición cuantitativa que permitan determinar si una persona ha incorporado nuevos conocimientos o actitudes luego de una movilidad.

En consecuencia, se hace necesaria la construcción de una herramienta que permita medir los efectos de la movilidad, de manera 
que se puedan realizar estudios al respecto así como revisar la relación entre movilidad y prejuicio moderno.

El presente artículo tiene por objeto estudiar la relación entre movilidad internacional y prejuicio, en la concepción de Pettigrew y Meertens (1995), para una muestra de españoles de nacimiento que se encontraran viviendo en Espańa en el momento de la evaluación y que tuvieran contacto con inmigrantes.

\section{CONTEXTUALIZACIÓN Y CONOCIMIENTO PREVIO}

\section{EMIGRACIÓN DE ESPAÑOLES AL EXTRANJERO}

En la figura 1 se observa la evolución de la emigración de españoles al extranjero discriminada por sexo, entre el 2008 y el 2013. En este último año, la emigración absoluta fue de 71.068 personas, representando un incremento de $48,44 \%$ en el periodo estudiado.
De los nacidos en España que migraron en el 2013, los hombres (53,11\%) representan un porcentaje levemente más alto frente a las mujeres (46,88\%). Igualmente, se destaca la concentración de la emigración entre las edades de 0 a 9 años, las cuales representan el $43,51 \%$ de españoles que salieron de su país durante el ańo de estudio. Los adultos entre 30 y 39 ańos le siguen en representatividad con un $17,72 \%$ del total.

Asimismo, según el Censo Electoral 2012, para ese año había 1.591.639 españoles en el extranjero (INE, 2012).

En lo que respecta a los datos de la población inmigrante en Espańa, a 2013 la población extranjera en el país representaba el 10,74 \% sobre el total, según datos del Padrón Municipal.

Por país de nacimiento, el 49,45\% de los extranjeros presentes pertenecen a países europeos, seguido de los continentes americano $(25,96 \%)$ y africano $(17,77 \%)^{1}$.

FIGURA 1. EMIGRACIÓN DE ESPAÑOLES DE NACIMIENTO AL EXTRANJERO, 2008-2013

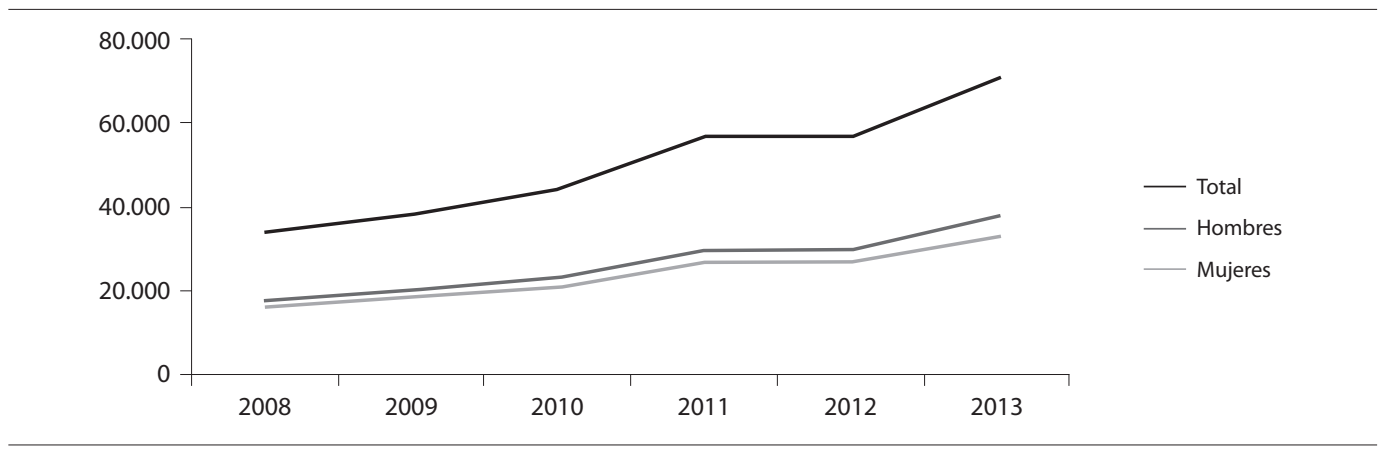

Fuente: elaboración propia a partir de datos del Instituto Nacional de Estadística (INE).

\footnotetext{
1 INE, Datos del Padrón municipal a primero de enero de 2014, consultados el 2 de marzo de 2015 en http://www. ine.es/jaxi/tabla.do
} 
Una vez presentados brevemente los datos sobre la emigración de espańoles al exterior y la situación de la inmigración en España, se procederá a realizar un recorrido teórico sobre el capital de movilidad y el prejuicio, para continuar con la exposición de la metodología y los resultados de la investigación.

\section{CAPITAL de MOVILIDAD}

El concepto de capital de movilidad debe ser diferenciado de varios homónimos utilizados en las ciencias sociales. Primero, como capital de movilidad suele denominarse también a las posibilidades y capacidades que tiene un individuo de moverse espacialmente en su ciudad, es decir que es un término ligado a la movilidad urbana, fenómeno estudiado por Chevrier y Juger (2004), Le Breton (2004) y Jacques Levy (Allemand, 2004), entre otros.

Por su parte, la movilidad internacional del capital hace referencia al proceso por medio del cual el capital se mueve libremente de un país a otro, en función del análisis que realizan los actores del sistema sobre las políticas macroeconómicas, las tasas netas de rentabilidad, la calificación de la economía local, entre otros. Por tanto, suele ser usado en el campo de la Economía y las Finanzas Internacionales para indicar el grado de apertura financiera que existe en un país (Veiga, 2012).

Otras formas similares de denominar al capital de movilidad han sido: capital espacial (Garneau, 2006; Levy y Lussault, 2003; Terrier, 2009), capital geográfico (Lazzarotti, 2006), competencias migratorias (Ballatore, 2007), competencias geográficas (Ceriani, Knafou y Stock, 2004; Lazzarotti, 2006), capital migratorio (Hanus, 2009; Ballatore y Bloss, 2008; Erlich, 2012; Yequin, 2009), motilidad (Kaufmann, 2005), saber migrar (Allemand, 2004; Simon, 2008); capital social internacional (Wagner, 1998), capital humano transnacional (Changgui et al., 2004) o capital de movilidad geográfico (Cailly, 2004). Sin embargo, estas denominaciones no distinguen el carácter interno/internacional ni otras particularidades encontradas por los autores.

En efecto, con base en el desarrollo teórico sobre las formas del capital realizado por Bourdieu (1986), son varios los autores que han conceptualizado diferentes tipos de capital que describen la trayectoria de movilidad de los individuos. Dado que existen varias acepciones de capital de movilidad, así como las variables que lo componen, se expondrá posteriormente la que se ha tomado como base para el presente artículo.

\section{Capital de movilidad según Murphy-Lejeune (2013)}

Murphy-Lejeune (2003, 2013) señala que el capital de movilidad es un componente del capital humano, que permite a los individuos aumentar sus competencias como consecuencia de su estancia en el extranjero. Asimismo, lo considera complementario del capital cultural propuesto por Pierre Bourdieu.

En ese sentido, la autora lo describe como "aquel capital que sirve tanto de punto de partida como el que se acumula" (Murphy-Lejeune, 2003, p. 46), e identifica como variables del capital de movilidad: los vínculos familiares con el extranjero, la duración y diversidad de contactos y estancias, y la experiencia anterior 
de adaptación. También se pueden incluir las competencias lingüísticas, la familiaridad con diversas culturas y los vínculos personales con el extranjero (Murphy-Lejeune, 2001, p. 158).

En conclusión, el capital de movilidad es reconocido por la comunidad científica, la cual ha utilizado diversas acepciones para hacer referencia a los recursos con que cuenta una persona para movilizarse.

Para los efectos del presente estudio, el capital de movilidad se entenderá como los recursos adquiridos por una persona durante su trayectoria vital, los cuales indican tanto la posibilidad de desplazamiento al exterior como la movilidad geográfica (sea real o imaginaria) realizada. Dicho capital genera a su vez nuevas formas de patrimonio, en virtud de las habilidades y competencias generadas.

\section{EFECTOS DE LA MOVILIDAD}

En su estudio sobre capital de movilidad en estudiantes, Murphy-Lejeune (2013) reconoce el "aprendizaje intercultural" que pudieron haber tenido los estudiantes durante su estancia en el exterior, atribuido a una mayor interacción con nativos, aprendizaje de la nueva cultura, interacciones con otros extranjeros y otras experiencias adquiridas, como el mejoramiento en lengua extranjera, por ejemplo.

Para ello, demanda a los participantes aspectos como la inserción en la cultura de acogida (Murphy-Lejeune, 2013, p. 217). Asimismo, pregunta a los participantes cuál creen ellos que fue su progreso en los referidos aspectos una vez terminada la movilidad, reconociendo que la misma tiene un efecto sobre el individuo.
En suma, Murphy-Lejeune (2003) señala la relación entre el capital de movilidad y el prejuicio, al reconocer como efecto positivo de la estancia en el exterior, un aumento en las relaciones con extranjeros una vez el estudiante retorna a su país de origen. Así, "la conciencia intercultural se enriquece, lo cual desafía los prejuicios y estereotipos, generando efectos una vez producido el retorno" (p. 83).

Otros aspectos señalados por los estudiantes tienen que ver con una mayor apertura, mejora en las competencias de comunicación y menores barreras mentales en el reencuentro con el otro.

En el mismo sentido que Murphy-Lejeune (2013), García y Gómez (1998) en su estudio sobre el efecto del turismo en el cambio de actitudes para una muestra de españoles que han viajado a Italia, concluyen que tras el viaje, los participantes valoran de forma más positiva la sociedad italiana.

Anastasopoulos (1992), por el contrario, refiere un impacto negativo del efecto de la movilidad en su estudio sobre una muestra de griegos que viajaron a Turquía, en lo que pensaban sobre la calidad de vida, las instituciones y los aspectos culturales turcos.

De otro lado, Jafari, Milman y Pizam (1991), en su estudio sobre estudiantes estadounidenses que viajaron a la antigua Unión Soviética, mostraron que la experiencia turística no cambió sus actitudes y opiniones sobre dicho país y los soviéticos en general.

Para la elaboración del instrumento de medición de los efectos de movilidad se tuvieron en cuenta las variables identificadas por Murphy-Lejeune (2001, 2003, 2013), 
así como los aportes en términos de contacto intergrupal desarrollados por Allport (1962) y Pettigrew y Meertens (1995).

\section{Prejuicio}

Allport definió el prejuicio como "una antipatía que se apoya en una generalización imperfecta e inflexible. Puede sentirse o expresarse. Puede estar dirigida hacia un grupo en general, o hacia un individuo por el hecho de ser miembro del grupo" (1962, p. 24).

Dovidio y Gaetner (1986), por su parte, realizan un compendio teórico sobre investigaciones en materia del prejuicio, incluyendo -entre otros- los trabajos de Hass, Katz y Wackenhut (1986) y el racismo ambivalente, el racismo moderno desarrollado por $\mathrm{McCo}$ nahay (1986) y la forma aversiva del racismo desarrollada por los mismos editores. Los anteriores aspectos serán retomados por Pettigrew y Meertens (1995) en su estudio sobre las nuevas formas del prejuicio.

Así, Pettigrew y Meertens (1995) encontraron dos tipos diferentes de prejuicio que pueden existir en la población, a saber: prejuicio manifiesto y prejuicio sutil. El primero, mucho más cercano, hostil y directo, y, el segundo, más distante, frío e indirecto.

Los investigadores demostraron que tanto el prejuicio manifiesto como el sutil forman cada uno dos factores correlacionados que hacen parte de un constructo superior (el prejuicio).

Los hallazgos soportan la teoría del contacto intergrupal, en tanto la amistad explica las puntuaciones en prejuicio manifiesto y sutil, teniendo bajo control otros predictores.
Un aporte interesante de la investigación sobre el prejuicio desarrollada por Pettigrew y Meertens (1995), es la tipología de los participantes realizada según su puntuación en ambas escalas: fanáticos, sutiles, igualitarios y sujetos tipo error (tabla 1).

TABLA 1. TIPOLOGÍA DE PREJUICIO SEGÚN PUNTUACIÓN EN ESCALAS DE PETTIGREW $Y$ MEERTENS (1995)

\begin{tabular}{|c|c|c|c|}
\hline \multicolumn{2}{|c|}{ Escalas } & \multicolumn{2}{c|}{ Prejuicio sutil } \\
\cline { 3 - 4 } & Bajo & Alto \\
\hline \multirow{2}{*}{$\begin{array}{c}\text { Prejuicio } \\
\text { manifiesto }\end{array}$} & Alto & Error & Fanáticos \\
\cline { 2 - 4 } & Bajo & Igualitarios & Sutiles \\
\hline
\end{tabular}

Fuente: Pettigrew y Meertens (1995, p. 69).

En el presente estudio se analiza el concepto de prejuicio moderno tal y como es concebido por Pettigrew y Meertens (1995), reconociendo que junto con el aspecto manifiesto, abierto y tradicionalmente considerado racista, se presenta también una nueva forma de prejuicio que estaría basada en la exageración de las diferencias culturales, la ausencia de emociones positivas (en contraposición a la expresión de emociones negativas del racismo antiguo) y la defensa de valores tradicionales del endogrupo.

Las variables y el instrumento por utilizar serán las escalas de prejuicio manifiesto y prejuicio sutil desarrolladas por Pettigrew y Meertens (1995), las cuales se expondrán en el apartado de instrumentos.

\section{TEORÍA DEL CONTACTO Y EFECTOS DE LA MOVILIDAD}

En su estudio sobre el prejuicio, Allport (1962) identificó el contacto como uno de los factores 
que podía incidir en la disminución de los prejuicios hacia los grupos minoritarios.

Para ello, el autor plantea que se deben presentar varios supuestos para que el contacto tenga un efecto sobre el prejuicio: igual estatus social, mismas metas, cooperación intergrupal y apoyo a la legalidad.

Basados en Allport (1962), Christ, Pettigrew, Tropp y Wagner (2011), Pettigrew (1998, 2008) y Pettigrew y Tropp (2008) han estudiado el efecto del contacto intergrupal en la reducción del prejuicio, y señalan que la dirección del contacto al prejuicio es mayor que del prejuicio al contacto (Pettigrew, 2008, p. 188); de la misma manera, encuentran que los supuestos del contacto de Allport (1962) son facilitadores y no elementos esenciales y ratifican que el contacto intergrupal reduce el prejuicio (Christ et al., 2011, pp. 271, 274).

Asimismo, Nesdale y Todd (2000), en su estudio experimental, hallaron un efecto positivo de la intervención intercultural realizada en los participantes, los cuales demostraron un aumento del conocimiento y la apertura cultural frente a otras nacionalidades.

Por supuesto, a la par de las investigaciones que han soportado la correlación negativa entre contacto intergrupal y prejuicio, existen las que proponen otros resultados. Por ejemplo, algunas situaciones de contacto pueden aumentar el prejuicio, si los miembros del endogrupo se sienten amenazados o si no eligieron el contacto, como en el lugar de trabajo, donde la competencia está presente (Christ et al., 2011, p. 277).

En lo que respecta al presente estudio, se retomarán los postulados de la teoría del contacto y los demás estudios que reconocen en la movilidad un cambio en las actitudes hacia otras culturas.

\section{Objetivos del PRESENTE ESTUdio}

El objetivo central del presente estudio es analizar la relación entre la movilidad internacional de los españoles y su opinión sobre el colectivo de inmigrantes en España. Para ello, se han planteado hipótesis de investigación que se detallan a continuación.

\section{HIPÓTESIS DE INVESTIGACIÓN}

Las hipótesis teóricas se formulan en sentido unidireccional, señalando los resultados que la literatura sugiere. Sin embargo, los análisis estadísticos se han realizado de forma bilateral (a dos colas), dada la naturaleza exploratoria del estudio.

A continuación se describen cada una de las hipótesis teóricas.

\section{EFECTOS DE LA MOVILIDAD}

- Los individuos con mayores efectos de movilidad presentarán menores niveles de prejuicio manifiesto y sutil.

\section{TEORÍA DEL CONTACTO}

- Los individuos que comparten más actividades con inmigrantes presentarán mayores efectos de movilidad.

- Los individuos que valoran positivamente su relación con inmigrantes presentarán mayores efectos de movilidad. 


\section{MÉTODO}

La presente es una investigación cuantitativa, no experimental, mediante un cuestionario con preguntas cerradas aplicado a una muestra de españoles de nacimiento residentes en Espańa en el momento de la evaluación y que, además, mantienen contacto con inmigrantes.

La herramienta de evaluación utilizada incluye instrumentos ya adaptados a la cultura española como el de Pettigrew y Meertens (1995), y se han elaborado instrumentos para medir la dimensión de los efectos de movilidad en función de los modelos teóricos que sustentan dicho constructo.

\section{Participantes}

En total, 441 personas respondieron el cuestionario, de las cuales fueron seleccionadas 259, puesto que las demás no cumplían los criterios de inclusión (ser españoles de nacimiento; vivir en España en el momento de la evaluación; tener contacto con inmigrantes y completar el cuestionario). De las 259 personas seleccionadas, fueron eliminadas 4 por pertenecer al grupo de error o de respuesta incoherente, tal y como señalan Pettigrew y Meertens (1995); es decir, son sujetos con puntuaciones altas en prejuicio manifiesto pero bajas en prejuicio sutil. La muestra final incluye 255 participantes.

El cuestionario fue enviado a más de 33 personas, solicitando su diligenciamiento y difusión entre otros españoles. Asimismo, fueron publicados enlaces de acceso al mismo, en 127 grupos/sitios de internet. No es posible calcular la tasa de respuesta, dado que se desconoce la población global que participa en los grupos de viajeros.

La edad de los participantes en la muestra final de 255, osciló entre los 18 y los 63 años $(\mathrm{M}=33,24$; $\mathrm{DT}=9,38)$, con una participación mayoritaria de mujeres $(58,4 \%)$ frente a hombres (41,6\%). La muestra no es probabilística.

\section{INSTRUMENTOS}

\section{Efectos de la movilidad}

Los efectos de la movilidad se midieron mediante 8 ítems, donde se les preguntaba a los participantes por su progreso después de la movilidad, en los siguientes aspectos: interacción con nativos, extranjeros, aprendizaje de otra lengua, opiniones sobre las migraciones internacionales, valoración de la cultura propia, empatía hacia culturas en el país de origen y mayor disposición a hacer amistad con personas de otras culturas. A las anteriores preguntas, los participantes podían contestar de uno a cinco, siendo uno (1) muy poco y cinco (5) mucho.

Esta escala estaba compuesta por cuatro ítems tomados textualmente de Murphy-Lejeune (2013), y el resto fueron redactados con base en los aportes sobre el contacto realizados por Allport (1962) y Pettigrew y Meertens (1995).

\section{Contacto con inmigrantes}

Para conocer si los participantes tenían contacto con inmigrantes, se les realizó una pregunta cerrada sobre si en su vecindario, lugar 
de trabajo o de estudios había personas inmigrantes. La respuesta posible era dicotómica y excluyente (sí, no).

Posteriormente, se les solicitaba que expresaran qué tanto compartían actividades con inmigrantes (algunas, bastantes, muchas) y cómo valoraban sus relaciones con este colectivo (afectiva, normal, neutra, poco afectiva).

Los ítems de este instrumento se han elaborado en función de los resultados aportados por la teoría del contacto y su relación con el prejuicio (Pettigrew, 1998; Frias-Navarro et al., 2013).

\section{Prejuicio manifiesto y prejuicio sutil}

Para medir el prejuicio moderno, se utilizó la Escala de Pettigrew y Meertens (1995) sobre Prejuicio Manifiesto y Sutil en la adaptación realizada por Frias-Navarro, Monterde i Bort y Peris (2009). Las puntuaciones posibles se presentaron en una escala tipo Likert entre 1 a 6 , siendo 1 totalmente en desacuerdo y 6 totalmente de acuerdo.

Asimismo, la elaboración de la tipología de Pettigrew y Meertens (1995) de sujetos igualitarios, sutiles y fanáticos, se llevó a cabo utilizando como punto de corte la puntuación de 27 en cada una de las dos subescalas -en el mismo sentido de la investigación desarrollada por Ramírez-March (2014) - y teniendo en cuenta que la muestra de estudio está altamente representada por individuos igualitarios.

\section{Procedimiento}

La metodología del presente estudio es no experimental, donde se pretende investigar la relación bidireccional entre los constructos efecto de la movilidad, la teoría del contacto y el prejuicio moderno.

\section{Recogida de la muestra}

La recogida de la muestra se llevó a cabo a través de redes sociales, grupos de viajeros y enlaces de acceso masivo. Para lo anterior, se utilizó el servicio prestado por Surveymonkey, con la creación de tres recopiladores: dos enlaces en página web y uno en Facebook. La toma de la muestra se llevó a cabo entre el 2 y el 26 de junio de 2014. Posterior a la depuración de los participantes, se procedió al ingreso de la información en el paquete estadístico spss.

\section{RESULTADOS}

Los resultados se exponen de la siguiente forma: inicialmente se presentan los estadísticos descriptivos relevantes de acuerdo con las hipótesis planteadas en en el apartado Hipótesis de la investigación.

Posteriormente, se describen las escalas utilizadas para medir los constructos (prejuicio manifiesto y prejuicio sutil, y efectos de la movilidad), los análisis de consistencia interna de esta última escala y los análisis de fiabilidad de la misma.

Por último, se detalla la comprobación estadística de las hipótesis planteadas, mediante un análisis de correlación entre los constructos, así como pruebas estadísticas para comparar si la diferencia de medias observada en los estadísticos descriptivos es estadísticamente significativa y permite concluir en el sentido de las hipótesis. 


\section{ESTADÍSTICOS DESCRIPTIVOS}

\section{Puntuaciones totales y estadísticos descriptivos de las variables analizadas}

Los estadísticos descriptivos para las variables de efectos de movilidad, prejuicio manifiesto y prejuicio sutil, pueden observarse en la tabla 2.

TABLA 2. ESTADÍSTICOS DESCRIPTIVOS DE LAS VARIABLES ANALIZADAS

\begin{tabular}{|c|c|c|c|c|c|c|}
\hline 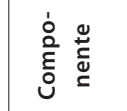 & $\begin{array}{l}\frac{0}{0} \\
\frac{0}{\frac{10}{2}} \\
\frac{7}{>}\end{array}$ & $\mathrm{N}$ & 站 & 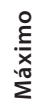 & $\begin{array}{l}\frac{\pi}{\bar{\sigma}} \\
\sum\end{array}$ & 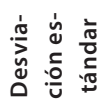 \\
\hline $\begin{array}{l}\text { Efectos de } \\
\text { la movili- } \\
\text { dad }\end{array}$ & $\begin{array}{l}\text { Efectos de } \\
\text { la movili- } \\
\text { dad }\end{array}$ & 249 & 11 & 40 & 30,89 & 6,112 \\
\hline \multirow{2}{*}{ Prejuicio } & $\begin{array}{l}\text { Prejuicio } \\
\text { sutil }\end{array}$ & 255 & 10 & 60 & 23,74 & 8,021 \\
\hline & $\begin{array}{l}\text { Prejuicio } \\
\text { manifiesto }\end{array}$ & 255 & 10 & 52 & 18,78 & 6,847 \\
\hline
\end{tabular}

A continuación, se detallan los resultados de los estadísticos descriptivos para cada una de las variables analizadas en función de la tipología creada por Pettigrew y Meertens (1995). Posteriormente, se analizarán las hipótesis teóricas mediante procedimientos de inferencia estadística.

\section{Efectos de la movilidad}

Las diferencias de medias en la medida de efectos del contacto se presentó en el siguiente orden: fanáticos $(\mathrm{M}=25,81 ; \mathrm{DT}=7,04)$, sutiles $(\mathrm{M}=31,16$; $\mathrm{DT}=6,55)$ e igualitarios $(\mathrm{M}=31,59 ; \mathrm{DT}=5,50)$.

\section{Contacto, valoración del contacto con inmigrantes y efectos de la movilidad}

Existen diferencias de medias entre los participantes que comparten algunas actividades con inmigrantes $(\mathrm{M}=29,95$; DT $=6,08)$ y los que comparten bastantes actividades $(\mathrm{M}=33,24$; $\mathrm{DT}=5,55)$.

Igualmente, los españoles que valoraron su relación con inmigrantes como normal, reportaron menores efectos de la movilidad $(\mathrm{M}=29,93 ; \mathrm{DT}=6,19)$, frente a quienes consideraron que su relación era afectiva $(\mathrm{M}=32,70 ; \mathrm{DT}=5,53)$.

\section{INSTRUMENTOS DE MEDIDA: FIABILIDAD Y CONSISTENCIA INTERNA}

\section{Prejuicio manifiesto y prejuicio sutil}

Se realizaron análisis de fiabilidad para las escalas de Prejuicio sutil y Prejuicio manifiesto, mediante la prueba estadística Alfa de Cronbach, encontrando consistencia interna tanto para Prejuicio sutil $(0,83)$ como para Prejuicio manifiesto $(0,79)$

\section{Efectos de la movilidad}

El análisis de fiabilidad del constructo Efectos de la movilidad arrojó consistencia interna de 0,86 mediante la prueba de Alfa de Cronbach. En la tabla 3 se detallan los resultados de fiabilidad si el ítem es eliminado de la escala.

Para estudiar la estructura interna de la escala, se realizó un análisis factorial exploratorio con el método de componentes principales (rotación varimax), en el que se encontró 
una solución de un factor. El conjunto de la varianza explicada fue de $53,15 \%$ en la escala.

TABLA 1. ANÁLISIS DE CONSISTENCIA INTERNA DE LA ESCALA DE EFECTOS DE MOVILIDAD

\begin{tabular}{|c|l|c|}
\hline Ítem & \multicolumn{1}{|c|}{ Afirmación } & $\begin{array}{c}\text { Alfa de Cronbach } \\
\text { si el elemento se } \\
\text { ha suprimido }\end{array}$ \\
\hline 1 & $\begin{array}{l}\text { Interacciones con nativos } \\
\text { (gente local del(os) país(es) } \\
\text { que visitó) }\end{array}$ & 0,832 \\
\hline 2 & $\begin{array}{l}\text { Conocimiento sobre culturas } \\
\text { de otros países }\end{array}$ & 0,846 \\
\hline 3 & $\begin{array}{l}\text { Interacciones con otros ex- } \\
\text { tranjeros }\end{array}$ & 0,827 \\
\hline 4 & Lengua extranjera & 0,858 \\
\hline 5 & $\begin{array}{l}\text { Opiniones más realistas sobre } \\
\text { los problemas sociales vincula- } \\
\text { dos a las migraciones }\end{array}$ & 0,836 \\
\hline 6 & $\begin{array}{l}\text { Mayor valoración de la cultura } \\
\text { ylas tradiciones de su país en } \\
\text { comparacion con otros países }\end{array}$ & 0,880 \\
\hline 7 & $\begin{array}{l}\text { Mayor empatía con el resto de } \\
\text { culturas que hay en su país }\end{array}$ & $\begin{array}{l}\text { Mayor disposición a hacer } \\
\text { amistades con personas de } \\
\text { otras culturas }\end{array}$ \\
\hline
\end{tabular}

\section{COMPROBACIÓN ESTADÍSTICA DE LAS HIPÓTESIS}

\section{Efectos de movilidad y prejuicio manifiesto y sutil}

(H1: los individuos con mayores efectos de movilidad presentarian menores niveles de prejuicio manifiesto y sutil).

Tanto la correlación entre efectos de movilidad y prejuicio manifiesto $(r=-0,32$; $\mathrm{p}<0,001)$, como entre efectos de movilidad y prejuicio sutil $(r=-0,39 ; \mathrm{p}<0,001)$ es esta- dísticamente significativa. Por tanto, se puede concluir que a mayores efectos de la movilidad, menor prejuicio manifiesto y sutil.

En lo referente a la diferencia de medias entre los grupos por tipología de prejuicio, la prueba ANOVA arrojó que las diferencias son estadísticamente significativas para la variable de efectos de movilidad $\left(\mathrm{F}_{(2,246)}=11,39 ; \mathrm{p}<\right.$ 0,$\left.001 ; \mathrm{n}_{\mathrm{p}}{ }^{2}=0,085\right)$.

La prueba de Tukey señaló que la diferencia de medias entre fanáticos e igualitarios es estadísticamente significativa $(\mathrm{p}<0,001)$. Asimismo, la diferencia de medias entre fanáticos y sutiles es estadísticamente significativa $(\mathrm{p}=0,001)$.

Por el contrario, no existen diferencias estadísticamente significativas entre igualitarios y sutiles $(p=0,905)$.

Lo anterior indica que los fanáticos reflejan menos efectos de la movilidad que los sutiles e igualitarios.

\section{Efectos de la movilidad y contacto con inmigrantes}

(H2: los individuos que comparten más actividades con inmigrantes presentarán mayores efectos de la movilidad).

Los resultados de la prueba de correlación de Pearson para la variable de efectos de movilidad y el compartir actividades con inmigrantes arrojó resultados positivos estadísticamente significativos $(r=0,24 ; \mathrm{p}<0,001)$. Es decir, a más efectos de la movilidad, se comparten más actividades con inmigrantes.

Igualmente, la prueba ANOvA entre grupos (a1 = algunas, $\mathrm{a} 2$ = bastantes) señaló que la diferencia de medias encontrada es 
estadísticamente significativa $\left(\mathrm{F}_{(1,247)}=15,57\right.$; $\left.\mathrm{p}<, 001 ; \mathrm{n}_{\mathrm{p}}{ }^{2}=0,059\right)$. Por tanto, los participantes que comparten bastantes actividades con inmigrantes puntúan más alto en efectos de la movilidad, frente a quienes comparten solo algunas actividades con este colectivo.

\section{Efectos de la movilidad y valoración del contacto con inmigrantes}

(H3: los individuos que valoran positivamente su relación con inmigrantes presentarán mayores efectos de movilidad).

El análisis de correlación mediante la prueba de Pearson para las variables de efectos de la movilidad y valoración de la relación con inmigrantes resultó estadísticamente significativo $(r=0,21 ; \mathrm{p}=0,001)$. Esto es, a mayores efectos de la movilidad, mejor valoración de la relación con inmigrantes.

De la misma manera, se encontró que la diferencia de medias entre grupos ( $\mathrm{a} 1$ = normal, a2 = afectiva), es estadísticamente significativa $\left(\mathrm{F}_{(1,247)}=12,03 ; \mathrm{p}=0,001 ; \mathrm{n}_{\mathrm{p}}{ }^{2}=0,046\right)$. Por tanto, quienes valoraron como afectiva su relación con inmigrantes reflejaron mayores efectos de la movilidad, frente a quienes consideraron que su relación con este colectivo era normal.

\section{DISCUSIÓN}

Acerca de la relación entre los efectos de la movilidad, la teoría del contacto y el prejuicio moderno, se puede señalar que los individuos fanáticos puntuaron más bajo en efectos de la movilidad, frente a los sutiles e igualitarios.
Por su parte, el contacto con inmigrantes y la valoración del mismo se comportó en la dirección esperada: a mayor (y mejor) contacto con inmigrantes mayores efectos de la movilidad.

A continuación se discuten los resultados en el mismo orden de las hipótesis planteadas. Al final, se rescatan los hallazgos más importantes de cara a futuras investigaciones y el aporte que el presente estudio realiza para el conocimiento de las relaciones intergrupales entre autóctonos e inmigrantes, en especial en lo referente a nociones como la movilidad internacional y sus efectos a nivel individual.

\section{EFECTOS DE LA MOVILIDAD Y PREJUICIO}

\section{Los individuos con mayores efectos de movilidad presentarían menores niveles de prejuicio manifiesto y sutil}

Los análisis de fiabilidad para la escala de efectos de la movilidad fueron bastante buenos y se correlacionaron negativamente tanto con el prejuicio manifiesto como con el sutil.

Además de respaldar lo propuesto por Murphy-Lejeune (2013) en torno a la existencia de efectos ocasionados por la movilidad, en particular en lo referente al progreso en interacciones, conocimiento y disposiciones frente al(os) país(es) visitado(s), la escala incluye aspectos detallados por Allport (1962) y Pettigrew y Meertens (1995) en cuanto al efecto del contacto sobre una revaloración del exogrupo y de la cultura propia. Asimismo, los sujetos fanáticos puntuaron más bajo en la escala de efectos de movilidad, frente a los sutiles e igualitarios. 
Estos hallazgos son concluyentes frente a la hipótesis planteada y corroboran las investigaciones sobre la teoría del contacto intergrupal desarrolladas por Allport (1962), Pettigrew (1998), Christ et al. (2011) y Pettigrew y Tropp (2008).

Igualmente, se encuentran en la misma dirección que los hallazgos de García y Gómez (1998) sobre los efectos de la movilidad en el cambio de actitudes hacia culturas de acogida, pero difieren de las investigaciones realizadas por Anastasopoulos (1992) y Jafari et al. (1991).

De otra parte, resulta interesante el caso de los sujetos sutiles, los cuales no reflejaron diferencias frente a los igualitarios en la escala de efectos de la movilidad.

¿Cómo se explican estos hallazgos? ¿Son los sutiles propensos a revelar progresos en términos de interacciones, mayores conocimientos y concientización sobre la situación de las migraciones, pero a la vez, incapaces de matizar las diferencias culturales en su país de origen?

¿Cómo sucede el proceso de reducción del prejuicio vía mayores efectos de la movilidad? O, en sentido opuesto, ¿cómo actúa el prejuicio en el (des)aprovechamiento de una movilidad internacional?

En suma, pese a los resultados observados en el grupo de los sutiles, y teniendo en cuenta los demás hallazgos, se puede concluir que la relación entre movilidad y prejuicio es negativa y que a mayores efectos de la movilidad, menores niveles de prejuicio (o viceversa).

No obstante, aun cuando se haya demostrado una generalización de los efectos de movilidad (interacciones con otras culturas) hacia el colectivo de inmigrantes en España, falta por estudiar cómo se ha producido esta generalización (sea esta situacional, grupal o hacia grupos no relacionados) y por qué se ha dado de manera diferenciada en los individuos.

\section{TEORÍ́ DEL CONTACTO}

\section{Los individuos que comparten más actividades con inmigrantes presentarán mayores efectos de la movilidad}

Los hallazgos permiten concluir que los individuos que comparten más actividades con inmigrantes puntúan más alto en cuanto a efectos de la movilidad, frente a quienes comparten algunas actividades.

Este hallazgo es sumamente importante y requiere que para investigaciones futuras se continúe indagando sobre la relación entre la movilidad internacional y la mayor disposición a interactuar con inmigrantes en el país de origen.

Lo anterior, para determinar si una mayor interacción con inmigrantes conduce a un deseo de interactuar con locales cuando se realiza una movilidad al exterior, o si por el contrario, el hecho de haber viajado al extranjero ocasiona que el individuo haya disminuido sus niveles de etnocentrismo y, en consecuencia, aumentado su interacción con inmigrantes.

\section{Los individuos que valoran positivamente su relación con inmigrantes presentarán mayores efectos de movilidad}

En el mismo sentido que la hipótesis 12, los individuos que valoraron como afectiva su relación con inmigrantes también puntuaron 
más alto en la escala de efectos de la movilidad, frente a quienes la valoraron como normal.

Nuevamente, lo que se plantea es si la movilidad es un mediador en el prejuicio, o, por el contrario, la tenencia de relaciones positivas con otras culturas en el país de origen incide en un mayor aprovechamiento del aprendizaje intercultural en los países visitados.

\section{ConCLusión}

En general, los hallazgos de la presente investigación permiten concluir que la movilidad internacional produce unos efectos diferenciados en los individuos, los cuales presentan varios patrones de relación con respecto al prejuicio manifiesto y sutil, lo que sugiere que se debe revisar la manera como son apropiadas las experiencias en el extranjero por parte de los individuos.

Del mismo modo, la puntuación de los sutiles en la escala de efectos de la movilidad sigue demostrando que se debe continuar con la investigación de este tipo de prejuicio (y de individuos), para mejorar la comprensión de la forma como se produce la perpetuación del prejuicio en quienes se consideran a sí mismos como no racistas, pero encuentran justificaciones para su rechazo hacia los miembros del exogrupo.

En cuanto a la teoría del contacto, sigue siendo un sustento teórico adecuado para estudiar las relaciones intergrupales, en especial si se tienen en cuenta los hallazgos de la presente investigación en torno a los efectos de la movilidad.

A través del anterior recorrido de los principales resultados de esta investigación, se pretende haber generado nuevos aportes en el estudio de las relaciones intergrupales derivadas del fenómeno migratorio y de movilidad humana en España.

Asimismo, se aportaron sugerencias en torno a nuevas líneas de investigación sobre la materia, para responder a una pregunta que subyace al tema de las relaciones intergrupales: ¿se puede vivir sin prejuicios?

\section{LIMITACIONES DEL ESTUDIO}

Una de las principales limitaciones del presente estudio está relacionada con el tipo de muestreo realizado (no probabilístico), el cual dificulta la generalización hacia toda la población española. Por tanto, se considera de suma importancia su posterior replicación en futuros estudios sobre el prejuicio hacia el colectivo inmigrante en España.

De otra parte, algunos de los participantes manifestaron sentirse incómodos al contestar la escala de Pettigrew y Meertens (1995), pues no podían diferenciar el tipo de inmigrante del que se estaba hablando. Ellos hubiesen preferido claridades del tipo: "inmigrante económico", o que la pregunta especificara el país de origen del inmigrante.

Lo anterior concuerda con la investigación llevada a cabo por Cuadrado, López y Navas (2012), que estudió la percepción de amenaza realista y simbólica de los españoles frente a tres colectivos de inmigrantes -ecuatorianos, marroquíes o rumanos-, encontrando diferencias estadísticamente significativas en los resultados.

De otra parte, en lo concerniente al contacto durante la estancia en el extranjero, el 
cuestionario no incluye una valoración del mismo por parte del participante, de manera que se pueda conocer si el contacto fue positivo o negativo. Adicionalmente, sería interesante conocer, por ejemplo, la frecuencia de los contactos.

Otra limitación del estudio es que no distingue una estancia larga de una corta por parte de los participantes, lo cual impide reducir la superficialidad de los contactos producidos por encuentros cortos como los viajes. El aspecto de la superficialidad es mencionado por Christ et al. (2011) en el metaanálisis realizado.

\section{CONCLUSIÓN FINAL}

La principal conclusión gira en torno al papel de los efectos de la movilidad, en su relación con el prejuicio moderno. Por tanto, mediante la creación del instrumento de medición de los efectos de la movilidad, se ha generado una herramienta que permite la mejor comprensión de la relación entre movilidad internacional y el prejuicio moderno.

En especial, se debe seguir estudiando esta relación para replicar la investigación y señalar si una mayor apropiación de experiencias en el extranjero por parte de los individuos se ve reflejada en menores niveles de prejuicio tanto manifiesto como sutil, tal y como lo sugieren los resultados del presente estudio.

De otra parte, se evidenciaron limitaciones del estudio y se sugirieron futuras investigaciones en torno a esta temática.

A partir de las principales conclusiones y del recorrido teórico realizado, se espera haber aportado nuevos conocimientos en esta área de las ciencias sociales y, sobre todo, nuevos inte- rrogantes por resolver en el camino inacabado de la investigación científica.

\section{REFERENCIAS}

Agulhon, C. y Xavier de Brito, A. (eds). (2010). Les étudiants étrangers à Paris. Entre affliation et repli. Paris: L'Harmattan.

Allemand, S. (2004). La mobilité comme capital. Sciences Humaines, I (145), 20-22.

Allport, G. (1962). La naturaleza del prejuicio. Buenos Aires: Editorial Universitaria de Buenos Aires, Rivadavia.

Anastasopoulos, P. G. (1992). Tourism and attitude change greek tourist visiting Turkey. Annals of tourism research, 19 (4), 629-642.

Ballatore, M. y Bloss, T. (2008). L'autre réalité du programme Erasmus: affinité selective entre établissements et reproduction sociale des étudians. Formation Emploi, 103, 57-74.

Ballatore, M. (2007). L'experience de mobilité des étudiants Erasmus: les usages inégalitaires d'un programme d'échange. Une comparaison Angleterre/France/Italie. Université Aix-Marseille et Turin, France - Italie. Recuperado de http:// hal.archives-ouvertes.fr/docs/00/20/47/95/PDF/ magalithese.pdf.

Bourdieu, P. (1986). The forms of capital. En Richardson, J. E. (ed.). Handbook of theory of research for the Sociology of Education (pp. 46-58). New York: Greenworld Presse.

Bredeloup, S. (2008). L'aventurier, une figure de la migration africaine. Cahiers AInternacionaux de Sociologie, 2 (125), 281-306.

Cailly, L. (2004). Pratiques spaciales, identités sociales et processus d'individualisation. Université François Rabelais de Tours, France. Recuperado de http:// 
tel.archives-ouvertes.fr/docs/00/39/79/01/PDF/

These_Cailly.pdf.

Castles, S. y Miller, M. (2009). The age of migrations: International Population Movements in the Modern World. New York: Palgrave Macmillan.

Ceriani, G., Knafou, R. y Stock, M. (2004). Les compétences cachées du touriste. Sciences Humaines, I (145), 28-30.

Changgui, C., Rosen, S. y Zweig, D. (2004). Globalization and transnational human capital: Overseas and returnee scholars to China. China Quarterly, 179, 735-757.

Chatterji, J. (2013). Dispositions and destinations: refugee agency and "mobility capital" in the bengal diaspora, 1947-2007. Comparative Studies in Society and History, 55 (2), 273-304.

Chevrier, S. y Juguet, S. (2004). Des voyageurs pas si bêtes. Sciences Humaines, I (145), 32-35.

Cuadrado, I., López, L. y Navas, M. (2012). Fiabilidad y evidencias de validez de la Escala de Percepción de Amenaza Exogrupal. Psicothema, 24 (3), 477-482.

Christ, O., Pettigrew, T., Tropp, L. y Wagner, U. (2011). Recent advances in intergroup contact theory. International Journal of Intercultural Relations, 35, 271-280.

Dervin, F. y Ljalikova, A. (eds.) (2008). Regards sur les mondes hypermobiles: mythes et réalités, I. Paris: L'Harmattan.

Dovidio, J. y Gaertner, S. (1986). The adversive form of racism. En Dovidio, J. y Gaetner, S. (eds.). Prejudice, discrimination, and racism (pp. 61-86). Orlando: Academic Press. Inc.

Erlich, V. (2012). Les mobilités étudiantes. Paris: La Documentation Française.

Erlich, V. (2013). Les mobilités étudiantes en Europe. Observatoire National de la Vie Étudiante, 28, $1-12$.
Frias-Navarro, D. (2009). Adaptación al español del instrumento de Prejuicio Manifiesto y Sutil de Pettigrew y Meertens (1995). Valencia: Universidad de Valencia. Recuperado de http://www.uv.es/ friasnav/PMPS.pdf.

Frias-Navarro, D., Barrientos-Delgado, J., Badenes-Ribera, L., Monterde-i-Bort, H. y Cárdenas-Castro, M. (2013). Sex differences in sexual prejudice. Contact as mediator variable. European Journal ofChild Development, Education and Psychopatho$\log y, 1,45-58$.

Frias-Navarro, D. y Soler, M. (2012). Prácticas del análisis factorial exploratorio (AFE) en la investigación sobre conducta del consumidor y marketing. Suma Psicológica, 19 (1), 47-58.

García, J. y Gómez, L. (1998). Influencia del turismo en el cambio de actitud: una perspectiva intergrupal. Revista de Psicología Social Aplicada, 8 (2), 5-20.

Garneau, S. (2006). Les mobilités internationales à la ére de la globalisation, Université Lumière-Lyon 2, France. Recuperado de theses.univ-lyon2.fr/ documents/getpart.php?id=9298action=pdf.

Glick, N. y Salazar, N. (2013). Regimes of mobility across the globe. Journal of ethnic and migration studies, 39 (2), 183-200.

Hanus, C. (2009). Être migrant, être sédentaire dans la petite sibérie française: une affaire de lignées familiales? Recherches familliales, 1 (6), 117-130.

Hass, G., Katz, I. y Wackenhut, J. (1986). Racial ambivalence, value duality and behavior. En Dovidio, J. y Gaetner, S. (eds.). Prejudice, discrimination and racism (pp. 35-57). Orlando: Academic Press. Inc.

Instituto Nacional de Estadística INE (2012). Censo electoral. Recuperado de www.ine.es

Instituto Nacional de Estadística INE (s.f.). Estadísticas del padrón continuo a 1ro de enero de 2014. Recuperado de www.ine.es 
Instituto Nacional de Estadística INE (s.f.). Flujo de emigración con destino al extranjero por año, sexo, grupo de edad, nacionalidad (española/ extranjera), país de nacimiento y país de destino. Recuperado de www.ine.es.

Jafari, J., Milman, A. y Pizam, A. (1991). Influence of tourism on attitudes: US students visiting USSR. Tourism Management, 12 (1), 47-54.

Knafou, R. (1998). La planète nomade: Les mobilités géographiques d'aujourd'hui. Paris: Belin.

Kaufmann, V. (2005). Mobilités et reversibilités: vers de sociétés plus fluides? Cahiers Internacionaux de Sociologie, 1 (118), 119-135.

Lazzarotti, O. (2006). Habiter: la condition géographique. Paris: Belin.

Le Breton, E. (2004). Vers un droit à la mobilité des plus pauvres? Sciences Humaines, I (145).

Levy, J. y Lussault, M. (2003). Dictionnaire de la Géographie. Paris, I.: Belín.

McConahay, J. (1986). Modern racism, ambivalence, and modern racism scale. En Dovidio, J. y Gaetner, S. (eds.). Prejudice, discrimination and racism (pp. 91-124). Orlando: Academic Press. Inc.

Murphy-Lejeune, E. (2000). Mobilité Internationale et adaptation interculturelle. Recherche et formation, 33, 11-26.

Murphy-Lejeune, E. (2001). Le capital de mobilité: genese d'un etudiant voyageur. Mélanges Crapel, 29, 137-165.

Murphy-Lejeune, E. (2003). Un portrait d'etudiants mobiles en l'an 2000: le cas français. En Vaniscotte, F., Houguenague A. y West, A. (eds.). La mobilité étudiante en Europe, mythe ou réalité? Comparaison France-Royaume Uni (pp. 39-90). Paris: L'Harmattan.

Murphy-Lejeune, E. (2013). L'étudiant européen voyageur. Un nouvel étranger. Paris: Didier.
Nesdale, D. y Todd, P. (2000). Effect of contact on intercultural acceptance: a field study. International Journal of Intercultural Relations, 24, 341-360.

Organización Internacional para las Migraciones (OIM) (2010). Informe sobre las Migraciones en el Mundo 2010. Recuperado de http://publications. iom.int/bookstore/free/WMR_2010_SPANISH.pdf.

Organización Mundial del Turismo (2013). Panorama омт del turismo internacional. Recuperado de http://dtxtq4w60xqpw.cloudfront.net/sites/all/ files/pdf/unwto_highlights13_sp_lr_0.pdf.

Pettigrew, T. y Meertens, R. (1995). Subtle and blatant prejudice in western Europe. European Journal of Social Psychology, 25, 57-75.

Pettigrew, T. (1998). Intergroup Contact Theory, Annual Review Psychology, 49, 65-85.

Pettigrew, T. y Tropp, L. (2008). How does intergroup contact reduce prejudice? Meta-analytic tests of three mediators. European Journal of Social Psychology, 38, 922-934.

Pettigrew, T. (2008). Future directions for intergroup contact theory and research. International Journal of Intercultural Relations, 32, 187-199.

Ramírez-March, A. (2014). Explorando las nuevas formas de prejuicio: la utilidad de las tipologías de prejuicio manifiesto y sutil [Trabajo fin de grado no publicado]. Valencia: Universidad de Valencia.

Rojas, M. (2008). Jóvenes inmigrantes y exclusión en Suecia. De la integración al conflicto. Cuadernos de Pensamiento Político, 165-195.

Simon, G. (2008). La planète migratoire dans la mondialisation. Paris: Armand Colin.

Stephan, C. y Stephan, W. (2000). An integrated treat theory of prejudice. Reduciing prejudice and discrimination, Mahwah. Psychology press, 23-47.

Tarrius, A. (1992): Les fourmis d'Europe: migrants riches, migrants pauvres et nouvelles villes internationals. Paris: L' Harmattan. 
Terrier, E. (2009). Mobilités et experiences territoriales des étudiants internationaux en Bretagne. Interroger le rapport mobilités spatiales/inégalités sociales à partir des migrations étudiantes. Université de Rennes 2, France. Recuperado de http:// tel.archives-ouvertes.fr/docs/00/45/81/98/pDF/ these'Terrier.pdf.

Veiga, L. (2012). El movimiento internacional de capitales. Revista de negocios del IEEM, 1, 76-77.
Wagner, A. (1998). Les nouvelles élites de la mondialisation: une immigration dorée en France. Paris: Presses Universitaires de France.

Wihtol de Wenden, C. (2001). Un essay de typologie des nouvelles mobilités. Hommes \& Migrations, $1233,5-12$.

Yequin, Z. (2009). Les illusions perdues d'un chinoise du nord à Belleville (enquête), Terrains \& Travaux, 2, 16, 195-2011. 